\title{
¡Chile despertó! Una investigación-acción en formación ciudadana de futuros docentes durante el estallido social*
}

\author{
Chile woke up! An action research in citizenship \\ education of future teachers during the social outbreak
Juan José Salinas Valdés ${ }^{\mathrm{a}}$, Jordi Castellví Mata ${ }^{\mathrm{b}}$ \& Pablo Camus Galleguillos ${ }^{\mathrm{c}}$
aUniversidad de Antofagasta, Antofagasta, Chile.凶juan.salinas.valdes@uantof.cl [orcid.org/0000-0003-3999-6231]
bUniversidad Internacional de La Rioja, Logroño, España. jordi.castellvimata@unir.net [orcid.org/0000-0002-6487-5477]
cUniversidad de Antofagasta, Antofagasta, Chile.pablo.camus@uantof.cl [orcid.org/0000-0001-6463-3892]

\section{RESUMEN}

El presente artículo expone una investigación-acción relativa a una propuesta didáctica denominada 'Módulo Cabildo Ciudadano', destinada a fortalecer las competencias ciudadanas y el empoderamiento constituyente de un grupo de futuros docentes de Educación Básica. Para ello, se abordaron el estallido social chileno ocurrido entre 2019 y 2020, así como el consiguiente proceso constitucional desatado, sucesos sincrónicos al desarrollo de las indagaciones. La valoración de la propuesta se realizó desde un enfoque mixto con predominio cualitativo. Se emplearon notas de campo, cuestionarios, entrevistas semiestructuradas y un grupo de discusión. La información obtenida fue procesada mediante un análisis cualitativo de contenido y otro de frecuencias descriptivas. Los resultados apuntan al potencial del abordaje de la realidad social para el desarrollo de competencias ciudadanas y del interés hacia los asuntos públicos. Asimismo, se determinaron una serie de fortalezas y debilidades, además de propuestas a considerar en el rediseño del módulo educativo.

PALABRAS CLAVE: educación ciudadana, formación de docentes, participación ciudadana, temas controversiales, estallido social.

\section{ABSTRACT}

The current paper shows the results obtained in an action research related to an educational intervention named 'Módulo Cabildo Ciudadano' (Module Citizen Council), aimed to strengthen

Investigación financiada por el proyecto El patrimonio, los problemas socialmente relevantes y la interculturalidad como herramientas didácticas para la FID, DE-766/2019, VRA, Universidad de Antofagasta. 


\section{J. J. Salinas et al.}

citizenship competences and constituent empowerment of a group of future Elementary Education teachers. To achieve it, we addressed the Chilean social outbreak occurred between 2019 and 2020 and the resulting constitutional process, both synchronic to the development of this research. The evaluation of the educational intervention was performed using a mixedmethods approach with the prevalence of a qualitative analysis. Hence, we utilized fieldnotes, questionnaires, semi-structured interviews and a discussion group. The data obtained was analyzed with qualitative content analysis and descriptive statistics. The results obtained reveal the potential of the treatment of current social problems to develop citizenship competences and interest in public issues. Moreover, we determined various strengths and weaknesses, and some proposals for the redesign of the educational module.

KEY WORDS: citizenship education, teacher education, citizen participation, controversial issues, social outbreak.

\section{INTRODUCCIÓN}

El Módulo Cabildo Ciudadano (MCC) constituye una propuesta en formación ciudadana cuyo objetivo educativo es fortalecer las competencias en ciudadanía de un grupo de futuros docentes de primaria, a la vez que incorpora el objetivo político-pedagógico, -en el sentido expresado por Freire (1999)-, de prepararlos para asumir su rol en el decisivo proceso constituyente que Chile vive desde el estallido social iniciado en octubre de 2019. Por tanto, el MCC se posiciona en la Pedagogía Crítica y su concepción de formación ciudadana para la participación activa y la búsqueda de la justicia social (Freire, 1997; Giroux, 2004).

El MCC se propuso el empoderamiento constituyente de los partícipes, es decir, su interés y disposición a participar, de forma activa e informada, en la discusión y elaboración de una nueva constitución para el país, a partir del análisis y discusión de problemas socialmente relevantes (Santisteban, 2019), en este caso, relativos al estallido social y al rol de la constitución de 1980 en la configuración de la realidad chilena.

Plantear el desarrollo de competencias ciudadanas puede ser un elemento estructurador de los aprendizajes a lograr. Sin embargo, esta propuesta buscó superar la lógica de las competencias instrumentales e individuales, y proponer unas alternativas, creadas a medida de este proyecto, centradas en la profundización de la democracia. En tal sentido, este artículo explora las posibilidades del uso de competencias ciudadanas, un concepto más ligado a la lógica instruccional propia de la Educación para la Ciudadanía (EpC), en proyectos más amplios de Formación Ciudadana (FC), a partir del tratamiento de problemas socialmente relevantes, del uso dialógico de la razón y del ejercicio público de la ciudadanía en el contexto de una comunidad democrática (Mesa \& Benjumea, 2011).

\section{¡CHILE DESPERTÓ! DE LA CRISIS NEOLIBERAL AL PROCESO CONSTITUYENTE}

El viernes 18 de octubre de 2019 comenzó en Chile el mayor estallido social de su historia, en lo que se configuró como una rebelión popular contra las condiciones de vida 
impuestas por uno de los legados de la dictadura de Pinochet al país: el sistema económico neoliberal. En palabras del historiador Gabriel Salazar (2020), se trató del: “...más grande y temible reventón social de toda la historia de Chile” (p.13). Uno de los primeros slogans gritado por los manifestantes fue ¡No son 30 pesos, son 30 años!, pues, si bien todo comenzó con protestas espontáneas contra el alza de 30 pesos en el pasaje del Metro de Santiago las cuales tuvieron un antecedente en los días previos con las evasiones masivas llevadas adelante por estudiantes secundarios-, en realidad sus motivos eran mucho más profundos: la ciudadanía protestaba en masa contra los efectos de las políticas económicas neoliberales en su calidad de vida; ;Chile despertó! Clamaron las y los chilenos desde redes sociales, ventanas, calles y plazas. A raíz de la represión, a mediados de marzo de 2020 los heridos y detenidos se contaban en miles, con cientos de denuncias de tortura, apremios ilegítimos y abusos sexuales (Instituto Nacional de Derechos Humanos, 2020), así como siete muertos.

Es posible identificar dos motivos centrales del estallido, ambos estrechamente relacionados entre sí. Primero, un sistema neoliberal que favorece el desinterés del Estado por garantizar los derechos sociales de la población, lo cual ha provocado elevados índices de desigualdad social y endeudamiento (Repetto, 2016), esto último incluso para satisfacer las necesidades más básicas, en lo que Aniñir \& Candina (2020) han definido como una violencia estructural del sistema. Segundo, a un sistema político, derivado de la constitución pinochetista de 1980, basado en una democracia restringida y tutelada (Grez, 2019). Ambos constituyen problemas históricos de arrastre (Salazar, 2020) que han tenido un papel central en dificultar el logro de los anhelos, tanto individuales como colectivos, de chilenas y chilenos.

La Constitución de 1980 establece el principio de subsidiaridad según el cual el Estado se restringe de participar en las actividades económicas, lo que en la práctica le ha llevado a desentenderse de asegurar el ejercicio de los derechos económicos, sociales y culturales de los ciudadanos, todo ello, pese a la ratificación en 1972 del pacto internacional respecto a ellos. Esto supone una profunda segregación de la población chilena según su capacidad de pago, por ejemplo, en las áreas de educación y salud. Esto conlleva un problema importante para la cohesión social, pues no solo implica la precarización de la calidad de vida de los grupos más vulnerables y los sectores medios, sino que también conduce a la guetificación social y espacial de una microminoría de clase alta (Morán, 2019). Al respecto, Heiss (2020) plantea que el 18 de octubre la ciudadanía chilena salió a las calles para protestar cansada de “(...) vivir con salarios de tercer mundo y con un costo de vida de país desarrollado; que el Estado abandone a su suerte a ancianos, enfermos y niños vulnerables; que una buena educación y una buena salud sean productos de lujo que solamente algunos pueden pagar" (p. 100).

La incapacidad del sistema político chileno para dar respuesta a las demandas sociales de la ciudadanía tiene su base fundamental en la Constitución de 1980, la cual fue elaborada y plebiscitada por la dictadura militar -en un proceso plagado de irregularidades- para perpetuar una concepción de la sociedad y de la economía de corte autoritario y neoliberal (Bassa, 2020). Para ello, sus creadores incorporaron una serie de elementos que garantizan la posibilidad de veto, por parte de la minoría de derecha, de cualquier reforma al sistema. Entre tales componentes se incluyeron: senadores designados -entre ellos luego estaría el mismo Pinochet-; quórums sumamente elevados para modificar la carta fundamental; un 


\section{J. J. SAlinas et al.}

sistema de elección binominal para impedir la entrada a cargos políticos de personas ajenas a las dos grandes coaliciones, ambas interesadas en mantener el neoliberalismo; el Consejo de Seguridad Nacional (COSENA), que posicionó a las fuerzas armadas como guardianes del sistema; y un Tribunal Constitucional (TC), con una buena parte de sus integrantes nombrados desde instancias de poder cooptadas por los partidos políticos tradicionales, por lo que en la práctica constituye una tercera Cámara no elegida democráticamente y con el poder de frenar el desarrollo legislativo de la agenda política pública (Bassa, 2020). Las diversas reformas a la Constitución de 1980 -siendo las principales las de 1989 y 2005- no han sido capaces de darle legitimidad frente a la ciudadanía, ni han sido lo suficientemente profundas como para permitirle canalizar las demandas de los chilenos.

El estallido social supuso un quiebre con la denominada 'subjetividad neoliberal', esto es, con la forma de pensar la sociedad según los principios de dicha ideología: individualismo, exaltación de los logros materiales, rechazo a lo comunitario, etc. (Flores \& García, 2011; Morán, 2019). La masividad y extensión de las protestas, que en algunas jornadas superaron el millón de personas solo en la capital, permitieron al imaginario colectivo tomar conciencia de que buena parte de los problemas, que se creían individuales, en realidad son colectivos, y fruto de un sistema económico y político concreto. De esta forma, no resulta extraño el nutrido respaldo ciudadano al desarrollo de una nueva constitución para el país en múltiples encuestas (Activa Research, 2019; CADEM, 2020), mientras que la sociedad parece haber entrado en un proceso de politización no visto desde el fin de la dictadura.

Desde el jueves 24 de octubre de 2019 la población autoorganizó 'cabildos ciudadanos' a lo largo de Chile, asambleas públicas para tratar la situación del país en el estallido, los motivos de este, el modelo político-económico imperante; y las características de una posible nueva constitución (Fuentes, 2019), constituyéndose, de esta forma, en instancias espontáneas de formación social y política para la ciudadanía. Tal tipo de autoorganización comunitaria tiene antecedentes en las asambleas ciudadanas y cabildos territoriales organizados por la población para enfrentar diversas medidas neoliberales -tales como alzas en insumos básicos o privatización de carreteras- durante el periodo 2010-2013, y en los llamados 'cabildos locales', organizados por el segundo gobierno de Michelle Bachelet en el contexto de su fallido intento por cambiar la constitución.

El desafío de desarrollar una nueva constitución dotada de legitimidad implica, como condición sine qua non, la necesidad de una amplia participación ciudadana, tanto en su aprobación como en el proceso de su elaboración, lo cual supone no solo rebasar los límites impuestos por el ‘Acuerdo por la Paz Social y la Nueva Constitución’1 a la soberanía constituyente popular, sino también con la necesidad de que la ciudadanía se reeduque a sí misma para asumir la tarea de crear una nueva carta fundamental (Heiss, 2020). En este sentido, Bassa (2020) sostiene que el éxito del proceso constituyente depende directamente de la participación popular, mientras que Salazar (2020) agrega que para lograr el pleno ejercicio de la soberanía constituyente: "La ciudadanía debe, pues, con urgencia, reeducarse a sí misma. El futuro de todos depende de esa auto-educación" (p.53). Nuestra disposición a contribuir a dicha tarea dio origen a la propuesta didáctica presentada en estas páginas.

Acuerdo alcanzado la madrugada del 15 de noviembre de 2019 por un amplio espectro de partidos políticos, pero sin participación ciudadana. 


\section{FORMACIÓN CIUDADANA Y FORMACIÓN INICIAL DOCENTE}

La Educación Ciudadana posee una importancia cada vez mayor en los sistemas educativos actuales, lo cual tiene su origen en la demanda de las sociedades por mayor participación en los asuntos públicos y en la necesidad de enfrentar los graves problemas sociales y ecológicos que, a diversas escalas, amenazan a la humanidad. En cuanto a la Educación Superior, resulta necesario prestar una especial atención a la Formación Inicial Docente (FID), debido al rol clave para la sociedad que cumple el profesorado en la tarea de educar ciudadanos. En este sentido, OREALC y UNESCO (2017) resaltan una serie de fortalezas de esta área en las universidades latinoamericanas, tales como su extendida presencia y una amplia adscripción a los principios democráticos, no obstante, advierten del predominio del enfoque teórico-expositivo al concretarla en las aulas y a su posición marginal en las áreas curriculares.

Las y los docentes reflejan en sus prácticas educativas sus ideas personales, sus nociones sobre la ciudadanía y la democracia, y sus visiones respecto a los objetivos de la educación (Thornton, 1991). En este sentido, las experiencias vivenciadas durante su etapa como estudiantes contribuyen ampliamente a construir tales ideas y, por tanto, inciden en la forma en como luego concretan su propio quehacer pedagógico. De esta forma, Reyes, Campos, Osandón y Muñoz, (2013) nos plantean, en un estudio empírico realizado con docentes chilenos de segundo ciclo de educación básica, que al planificar sus clases en ciudadanía estos consideran más sus propias experiencias ciudadanas que la formación teórica recibida en la universidad. Por ello, para formar a futuros formadores de ciudadanos y ciudadanas resulta necesario articular sus aprendizajes a partir de situaciones que impliquen el ejercicio real y profundo de la democracia, entendiendo que no es posible contribuir al desarrollo de la ciudadanía de otros sin antes haber desarrollado la propia.

Los enfoques que adopta la Educación Ciudadana en la actualidad son variados y, a menudo, se combinan en la construcción de los currículums oficiales. Una de las perspectivas predominantes tiene el objetivo de formar ciudadanos responsables (Westheimer \& Kahne, 2004) y está vinculada a la educación moral y cívica. Este enfoque suele ir acompañado de la promoción de símbolos e instituciones nacionales y de la cultura predominante, para desarrollar sentimientos e identidades patrióticas. También son comunes los enfoques que dan preeminencia a la educación financiera, a la creatividad, al emprendimiento y al desarrollo personal individual, para formar ciudadanos que vivan en contextos económicos que tienden a la desregularización, al individualismo y a la competitividad. Otros enfoques se plantean, desde los procesos educativos formales, el objetivo de educar a una ciudadanía participativa, que conozca la importancia de las instituciones democráticas y que esté dispuesta a defenderla (Kennedy, 2019), estos son comúnmente denominados como "Educación para la Ciudadanía" (Benjumea, Gutiérrez, Jaramillo, Mesa \& Pimienta, 2011). Finalmente, encontramos los enfoques relativos a la Pedagogía Crítica, interesados en el aprendizaje de la ciudadanía a lo largo de la vida y en diferentes contextos, y que usualmente son etiquetados bajo el concepto de "Formación 


\section{J. J. SAlinas et al.}

Ciudadana", estos defienden la necesidad de una ciudadanía crítica, participativa y transformadora, orientada hacia el bienestar colectivo y comprometida con un proyecto político de profundización de la democracia y la justicia social (Benjumea et al. 2011; Westheimer \& Kahne, 2004).

En los últimos años se ha tratado de construir en diversas latitudes, con mayor o menor éxito, una disciplina educativa desvinculada de la asignatura de ciencias sociales, historia y geografía, que aborde los retos a los que se enfrenta la ciudadanía en nuestros tiempos (Castellví \& González-Monfort, 2020). Dicho esfuerzo tiene su correlato en el caso chileno con la reciente introducción de la asignatura 'Educación Ciudadana', para los últimos dos niveles de Enseñanza Media (secundaria). En tal contexto, una de las aproximaciones que ha tenido más consenso institucional ha sido la del desarrollo de competencias ciudadanas, un concepto ligado frecuentemente al modelo de la Educación para la Ciudadanía (EpC) (Mesa \& Benjumea, 2011).

Instituciones como el Consejo de Europa (2018), la OREALC y la UNESCO (2017) reconocen la importancia de formar a una ciudadanía crítica y participativa que defienda los valores democráticos, la igualdad, la justicia social y los derechos humanos. De esta forma, a partir de 'competencias ciudadanas' (Zambrano, 2018), 'competencias para la ciudadanía' (Morillas, 2006), 'competencia educativa social y ciudadana' (Ortuño et al. 2011), o 'competencias para la cultura democrática' (Council of Europe, 2018) se ha construido un marco teórico en el cual se conciben una serie de valores, habilidades, actitudes, conocimientos y comprensiones críticas que configuran y operacionalizan dichos aprendizajes.

Más allá de sus diferencias internas, los enfoques relativos a competencias ciudadanas tienden a obviar, en el desarrollo de estas, el peso de la ideología y la opresión social en la generación de desigualdades y conflictos. En este sentido, Santisteban y Pagès (2007) consideran que la educación para una ciudadanía democrática se debe concebir como un proceso dinámico que sirva para conocer y cuestionar: “...las organizaciones sociales y políticas, su estructura como sistema de libertades, las formas de distribución del poder, de repartición de responsabilidades y de exigencia de criterios para la justicia y la igualdad" (p. 3). Así, estos autores afirman que es fundamental el desarrollo de una cultura política que incluya la configuración de las ideologías en base a las representaciones sociales de la población sobre la opinión pública, la capacidad de decisión y la participación política. De ello, surge una interrogante sobre la conveniencia de aplicar el enfoque de competencias en formación ciudadana, pero ¿esto es realmente así?

Una cuestión de primer orden pareciera ser determinar cuáles son las condiciones que permiten que actividades de EpC se desarrollen hasta constituirse en proyectos de formación ciudadana, y cuál es el papel del desarrollo de competencias en dicha tarea. En este sentido, Benjumea et al. (2011) sostienen que para ello se requiere introducir el principio de 'isegoría', es decir, la igualdad entre ciudadanos, que en educación se expresa -por ejemplo- en relaciones simétricas entre docentes y estudiantes. Además, y refiriéndose al caso específico de la educación superior, Mesa y Benjumea (2011) agregan la necesidad de practicar el uso público de la razón y de generar comunidades políticas que practiquen la democracia en el espacio común. 
Ahora bien, en propuestas de formación ciudadana es posible plantearnos el desarrollo de competencias como objetivos de aprendizajes concretos, que colaboren en la estructuración de las metas educativas de una experiencia o proyecto didáctico en particular. No obstante, resulta necesario superar su enfoque meramente instrumental/individual, para contextualizarlas bajo objetivos político-pedagógicos orientados a la profundización de la democracia (Freire, 1999). Para ello, se deben proponer competencias relativas a valores, habilidades y conocimientos propios de una sociedad de características democráticas avanzadas: igualdad, justicia social, interculturalidad, participación activa, pensamiento crítico, entre otras, y desarrollarlas en un ambiente de ejercicio real de la ciudadanía. Al respecto, la conveniencia de conciliar el concepto de 'competencias' con una formación ciudadana emancipadora, radica en su potencial para que las y los docentes -quienes se desempeñan en contextos educativos reales- puedan estructurar sus aprendizajes y, a la vez, avanzar hacia posiciones más políticas. Tal planteamiento se apoya en las palabras de Giruox (1997) cuando advierte que: "Para que la pedagogía radical se convierta en un proyecto político viable tiene que desarrollar un discurso que combine el lenguaje de la crítica con el lenguaje de la posibilidad" (p. 54). Ciertamente, una Pedagogía Crítica que ignore la realidad educativa no podrá incidir en ella.

Un elemento central que deben abordar los proyectos de formación ciudadana, que pretendan desarrollar competencias desde una perspectiva crítica, es el tratamiento de problemas socialmente relevantes presentes a diversas escalas. Al respecto, la bibliografía nos habla de 'Problemas Sociales Relevantes' (Santisteban, 2019), 'Controversial Issues' (Magendzo, 2016; Barton \& McCully, 2007) y de 'Questions Socialement Vives' (Legardez \& Simonneaux, 2006), tratándose de líneas de investigación que llegan a conclusiones similares. También lo hacen las líneas propias de las literacidades críticas, incorporando el trabajo a través de problemas sociales relevantes como una dimensión más de la formación del pensamiento crítico (Castellví, 2019). Al respecto, resulta necesario considerar que trabajar a partir de problemas sociales relevantes no significa problematizar el currículo, es decir, convertir en problemas los temas preestablecidos, sino abordar directamente cuestiones sociales candentes y de interés público.

Desde una perspectiva crítica, el docente no puede adoptar una postura neutral ante las problemáticas y conflictos que afectan al mundo, sino que debe dar testimonio de su propia posición y de la libertad de la ciudadanía para construir su propia opinión. Por otro lado, el objetivo nunca puede ser convencer o persuadir al alumnado de adquirir determinadas ideas, pues aquello se aleja de la intención de desarrollar su pensamiento crítico y constituye lo que Weiner (2008) califica como 'enseñanza pedante'. Evidentemente, aprender a pensar críticamente necesita de espacios para la formulación de las ideas propias. En línea con lo planteado, Freire (1999) señala:

(...) la total imposibilidad de ser neutrales frente al mundo, al futuro... nos plantea necesariamente el derecho y el deber de tomar una posición como educadores... -respetando el derecho que los educandos tienen de optar y aprender a optar, para lo cual necesitan libertad-... y jamás intentar imponerles nuestras opciones, subrepticiamente o no (p. 78). 


\section{J. J. SAlinas et al.}

La falta de espacios en la escuela y la universidad para abordar conflictos contemporáneos tiene un trasfondo en la concepción de la educación formal como un espacio libre de ideologías y de emociones (Estellés \& Castellví, 2020). De esta forma, las instituciones educativas se han configurado desde una apariencia de neutralidad y racionalidad impostadas, puesto que ningún conocimiento es libre de ideología y ninguna situación es libre de emociones (Castellví, Massip \& Pagès, 2019). Esta perspectiva, ha contribuido a la creación de una Educación Superior para el desarrollo de competencias que capacitan al alumnado para devenir en ciudadanos y ciudadanas que vivan en un contexto en el que impera una ideología hegemónica, el capitalismo neoliberal. En base a esta idea, los contextos educativos formales obvian hablar de ideologías -más que como un referente histórico- y educan una ciudadanía consumista y competente en lo productivo.

Ante la situación descrita, resulta necesario redefinir los programas universitarios para el desarrollo del pensamiento crítico de forma que devengan en una formación cultural y política para la ciudadanía, y para, como ha defendido Giroux (1997), incrementar las oportunidades de los grupos subordinados para participar activamente en la sociedad. Pues, desde este punto de vista, el objetivo de las ciencias sociales es que los ciudadanos entendamos, abordemos y solucionemos los problemas de nuestra sociedad, y, de esta forma, contribuir al fortalecimiento de la democracia. Para el caso de la Formación Inicial Docente, y para que los futuros profesores y profesoras puedan un día desarrollar adecuadamente la ciudadanía de sus propios alumnos y alumnas, resulta esencial que en su proceso formativo aborden la realidad social y experimenten acciones concretas y maximalistas de ejercicio ciudadano, en el contexto de comunidades democráticas abiertas a la reflexión, al diálogo y a la participación

El estallido social contra el neoliberalismo que Chile vivió entre octubre de 2019 y marzo de 2020, entendido como la mayor revuelta popular de su historia, es una oportunidad ineludible para formar ciudadanos y, asumiendo el momento histórico que vive el país, para empoderarlos con su pleno derecho a ser parte del proceso constituyente en marcha. En línea con tal idea, desde el estallido mismo se ha manifestado un elevado interés por crear secuencias educativas y materiales didácticos orientados a tratar sus causas, características y consecuencias (Hoecker \& Dardel, 2019; Sáez-Rosenkranz, 2019), la propuesta didáctica resultado de esta investigación-acción se suma a ellas.

\section{METODOLOGÍA}

El estudio presentado corresponde a una investigación-acción destinada a la mejora de la propia práctica docente (Latorre, 2016) y fue desarrollada con el objetivo de evaluar un módulo didáctico, basado en la realidad social, para el desarrollo de competencias ciudadanas y el interés por el proceso constituyente de futuros docentes de Educación Básica. Para ello, y desde un enfoque metodológico mixto con predominio cualitativo, se analizaron una serie de factores presentes en la valoración del alumnado y en la propia observación a partir de notas de campo (ver Tabla 1). 


\section{¡CHILE DESPERTÓ!}

Tabla 1. Factores considerados.

\begin{tabular}{cc}
\hline VALORACIÓN DEL ALUMNADO & OBSERVACIÓN \\
\hline Aprendizajes logrados & Competencias ciudadanas desplegadas \\
\hline Interés respecto al estallido social y el proceso constituyente & Motivación \\
\hline Fortalezas, debilidades y propuestas respecto al MCC & Productos generados \\
\hline Aporte a la FID & Factores emergentes \\
\hline
\end{tabular}

Fuente: Elaboración propia.

Primeramente, y en base a la literatura especializada en la materia (Magendzo, 2016; Santisteban, 2019) y a nuestras experiencias previas de formación ciudadana en educación superior (Salinas, 2019, 2020), se estableció la hipótesis-acción de que un enfoque centrado tanto en la participación democrática del alumnado como en el análisis y debate de la realidad social resultaría útil para el desarrollo de sus competencias ciudadanas y de su interés por el proceso constituyente. Tras ello, se determinó un diseño de indagación provisto de las siguientes fases: 1) diagnóstico sobre los conocimientos e ideas previas de los jóvenes respecto al estallido social y el proceso constituyente, 2) diseño y aplicación del MCC, 3) recolección y sistematización de la información, y 4) análisis e interpretación crítica de los resultados, con el objeto de determinar fortalezas, debilidades y propuestas a considerar en su rediseño.

Los partícipes de esta investigación fueron veintidós jóvenes de cuarto semestre de la carrera de Pedagogía en Educación Básica con Menciones (PEB), de la Universidad de Antofagasta. De ellos, el 77,3\% corresponde a mujeres y el 22,7\% a hombres, con un promedio de edad de 21,4 años al momento de las indagaciones. La gran mayoría posee residencia en las ciudades de Antofagasta (73\%) y Calama (14\%), mientras que el resto se distribuye homogéneamente en otros asentamientos de la misma región: Tocopilla, Taltal y Mejillones.

Durante el desarrollo del módulo se tomaron notas de campo que registraron las observaciones del docente-investigador, y una vez este finalizó se aplicó un cuestionario con preguntas abiertas y cerradas. Posteriormente, el análisis de los resultados obtenidos permitió determinar informantes clave respecto a los diversos factores indagados, los cuales luego participaron de cuatro entrevistas personales semiestructuradas y un grupo de discusión con otros cinco jóvenes. Los instrumentos utilizados fueron validados por el juicio de tres expertos, dos pertenecientes al área de la enseñanza de las Ciencias Sociales, y un tercero del campo de la Sociología Educativa.

La información obtenida fue procesada, en una primera instancia, mediante un análisis cualitativo de contenido (Massot, Dorio \& Sabarriego, 2016), para lo cual fue empleado el programa Atlas.ti 8. En esta etapa, la unidad de análisis considerada fueron las diversas referencias contenidas en las respuestas de los partícipes, entendidas como unidades de significado que articulan los diversos temas indagados (Hernández y Mendoza, 2018), lo que permitió codificar y categorizar inductivamente los datos recabados.

Una vez realizado el análisis de contenido, los datos correspondientes a preguntas cerradas del cuestionario (relativas al interés por el proceso vivido en Chile y el nivel de satisfacción con el módulo realizado) y aquellos de orden sociodemográfico fueron procesados 


\section{J. J. Salinas et al.}

utilizando el programa SPSS, con el objetivo de determinar frecuencias descriptivas expresadas en porcentajes-, y relaciones cruzadas entre las mismas. En este caso, la unidad de análisis utilizada fue cada individuo.

\section{DISEÑO E IMPLEMENTACIÓN DEL MÓDULO CABILDO CIUDADANO}

El estallido social que remeció al país desde el 18 de octubre de 2019 supuso la suspensión de clases tanto en el sistema escolar como en la Educación Superior. De esta forma, la asignatura de PEB denominada Formación Ciudadana, en la cual fue desarrollado el MCC, fue detenida temporalmente hasta enero de $2020 \mathrm{y}$, tras el receso estival de febrero, se extendió hasta mediados de marzo. No obstante, la intensificación de las protestas en Antofagasta, incluyendo cortes de múltiples rutas y la consiguiente paralización de la ciudad, implicó nuevamente la detención de las actividades educativas presenciales, por lo que fue necesario realizar la última sesión en forma telemática.

El estallido social supuso el reto de rediseñar sobre la marcha la planificación de la asignatura, pues resultaba del todo contradictorio no tratar un proceso de la mayor trascendencia para la formación ciudadana del alumnado. Además, implicó el reto de tratar acontecimientos que sucedían en tiempo real y en un clima de fuerte confrontación y polarización política, que se expresaba en las calles, redes sociales, medios de comunicación e, incluso, en las mismas instituciones del poder. Sin embargo, en aquel clima de inseguridad y continuas denuncias de violaciones a los DDHH, formar ciudadanía democrática se hacía más necesario que nunca.

El MCC fue diseñado para buscar la participación del alumnado y entregarle el protagonismo del proceso de aprendizaje. Por otro lado, trató directamente los acontecimientos que se venían sucediendo en el país desde el 18 de octubre, entendidos como problemas socialmente relevantes y/o temas controvertidos, a modo de utilizarlos como catalizadores para el desarrollo de competencias ciudadanas. Por su parte, el docente se posicionó políticamente en el respeto irrestricto de la democracia, la justicia social y los derechos humanos, a la vez que resaltó constantemente la importancia tanto de comunicar nuestras posiciones como de respetar el derecho de los demás a pensar distinto. Tomando en cuenta lo señalado, se desarrolló una propuesta didáctica basada en un diagnóstico inicial y tres etapas, resumidas en la Tabla 2.

Para levantar un diagnóstico inicial se solicitó a los jóvenes, en un breve informe, relacionar los sucesos acaecidos en el contexto del estallido social con diversos temas ya tratados en la asignatura: tipos de democracia, nociones para comprender la ciudadanía, sistemas económicos y las diversas generaciones de derechos humanos. Además, se les pidió realizar propuestas para la superación de la crisis.

Los resultados del diagnóstico apuntaron a que el total de jóvenes partícipes poseían abundantes conocimientos e ideas respecto al estallido en marcha. De igual forma, fueron capaces de interrelacionar adecuadamente los temas tratados previamente en la asignatura con los sucesos presentes. En este sentido, la mayoría situó las causas de la revuelta en la búsqueda de la ciudadanía chilena por mejorar sus condiciones de vida, desafiando las 
¡CHILE DESPERTÓ!

Tabla 2. Etapas del Módulo Cabildo Ciudadano.

\begin{tabular}{|c|c|c|}
\hline ETAPA & COMPETENCIAS POR FORTALECER & EJECUCIÓN \\
\hline Diagnóstico inicial & $\begin{array}{l}\text { Aplicar conceptos de política, ley y DDHH a la propia } \\
\text { realidad social }\end{array}$ & Informe \\
\hline 1. Aproximación al estallido social & \multirow{3}{*}{$\begin{array}{l}\text { Comunicar desde una estructura dialógica y tolerante } \\
\text { Valorar el derecho a pensar distinto } \\
\text { Valorar la dignidad y los derechos humanos } \\
\text { Valorar la democracia, la justicia social y la equidad } \\
\text { Contrastar críticamente las fuentes de información } \\
\text { Participar crítica y creativamente como ciudadanos } \\
\text { activos y éticamente responsables }\end{array}$} & $\begin{array}{l}\text { Clases } 1 \text { y } 2 \\
\text { (180 minutos) }\end{array}$ \\
\hline $\begin{array}{l}\text { 2. Habilidades y valores para un } \\
\text { debate ciudadano }\end{array}$ & & $\begin{array}{c}\text { Clase } 3 \\
\text { (90 minutos) }\end{array}$ \\
\hline $\begin{array}{l}\text { 3. Proceso constituyente: análisis y } \\
\text { propuestas }\end{array}$ & & $\begin{array}{l}\text { Clases } 4,5 \text { y } 6 \\
\text { (270 minutos) }\end{array}$ \\
\hline
\end{tabular}

Fuente: Elaboración propia en base a Council of Europe (2018) y Morillas (2006).

limitaciones al bienestar social colectivo impuestas por el modelo neoliberal, lo cual fue asociado a un ejercicio maximalista y comunitarista de la participación ciudadana. Por otro lado, respecto a los derechos humanos, hubo una tendencia mayoritaria a asociarlos con las graves imágenes de violencia policial que, en aquellos días, y a través de las redes sociales, impactaban a la audiencia nacional e internacional, mientras que fueron escasamente relacionados con otros derechos de orden político, económico, social y cultural.

Los cabildos ciudadanos que durante el estallido fueron organizados espontáneamente por la ciudadanía a lo largo de todo Chile tuvieron un carácter público y abierto. Por ello, las clases del módulo, excepto la primera y la última, fueron desarrolladas en espacios abiertos de la universidad, tanto en la llamada 'Plaza de la Reforma', centro cívico de la casa de estudios, como en el sector de 'Los Pastos', uno de los pocos lugares con césped y árboles en aquel desértico paisaje. Por el mismo motivo, se utilizó un parlante portátil con dos micrófonos inalámbricos con el objetivo de que las discusiones fuesen no solo audibles por el grupo curso, sino también, eventualmente, por todo miembro de la comunidad universitaria.

Etapa 1. En la primera y la segunda clase se buscó fortalecer el conjunto de competencias explicitadas en la Tabla 2 para esta fase. Para ello, se conformaron grupos de cuatro a cinco jóvenes, quienes, previa discusión interna, eligieron uno de los cuatro ejes introductorios: 1) Antecedentes del estallido social, 2) ¿Cómo se ha producido? Características, 3) Violaciones a los DDHH, y 4) Proceso constituyente. Estos fueron profundizados mediante la 'Guía 1: El derecho a pensar distinto' y el uso de una serie de códigos QR con información escrita y audiovisual que, en todo caso, fue posible complementar libremente utilizando los teléfonos móviles. Ambas clases finalizaron con discusiones a nivel de curso respecto a los temas tratados. Todas las guías y códigos QR utilizados en el MCC pueden ser consultados en la Fig. 1.

Etapa 2. En la tercera clase se buscó sobre todo fortalecer habilidades y valores de orden comunicativo, mediante el contraste crítico del tratamiento que dos medios de comunicación (uno de carácter internacional y otro propiedad de un conocido empresario chileno) hicieron de un mismo tema candente que por esos días copaba las portadas: el sabotaje por parte del alumnado de Enseñanza Media (secundaria) de la Prueba de Selección Universitaria (PSU), denunciada por estos como un elemento que contribuye a la reproducción de las desigualdades sociales del país. Reunidos en grupos, la problemática fue analizada a 


\section{J. J. Salinas et al.}

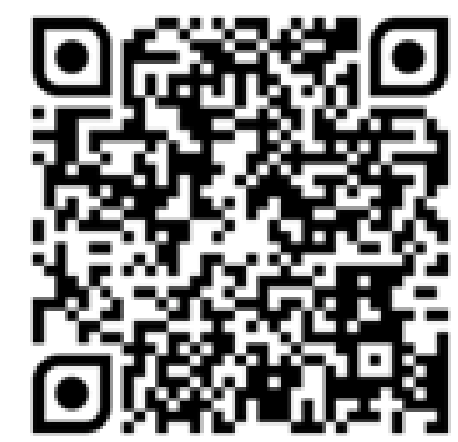

Fig. 1. Código QR. Material utilizado en el Módulo Cabildo Ciudadano.

Fuente: Elaboración propia.

partir de la 'Guía 2: Nosotros frente a los medios' y códigos QR con noticias en formato audiovisual, para luego discutir las respuestas en asamblea. Tal actividad estuvo orientada al desarrollo de habilidades críticas para la búsqueda, selección y análisis de la información. En este sentido, y a modo de ejemplo, en una de las guías del alumnado se puede leer:

Cada línea editorial tiene su propia ideología establecida, las cuales se enfocan en ciertos parámetros que se deben regir para informar los hechos noticiosos y así lograr causar indirectamente en la audiencia cierta postura ante el suceso ocurrido, esto si lo relacionamos con el tema PSU, veremos que existen diferentes enfoques relacionado con el mismo hecho (Grupo IV).

Etapa 3. En la cuarta y la quinta clase se trabajaron especialmente habilidades comunicativas y para la participación crítica y creativa de los futuros docentes, utilizando como tema catalizador el proceso constituyente en curso. En un primer momento, trabajaron grupalmente la ‘Guía 3. ¿Qué puede cambiar una nueva constitución?’, utilizando como apoyo el texto del mismo nombre del abogado Pedro Mujica (2019), miembro de la Fundación Educación y Ciudadanía. En la clase siguiente se utilizó la ‘Guía 4. ¿Analizamos la Constitución de 1980?’, en la cual se abordaron los siguientes aspectos: su estructura, la falta de reconocimiento de los pueblos indígenas, su abordaje de los derechos políticos, sociales y económicos, el rol subsidiario del Estado y el uso de recursos naturales y bienes de uso público, especialmente del agua, en atención a la grave problemática social provocada por la sequía y agravada por el dominio privado de los derechos de agua en Chile. Al final de ambas sesiones las respuestas grupales fueron puestas en común y discutidas por el conjunto del grupo curso.

En la sexta clase fue trabajada la ‘Guía 5. Propuestas para una nueva constitución’, en ella, el alumnado comparó lo estipulado por la Constitución chilena de 1980 con las constituciones de México, en materia de reconocimiento de pueblos indígenas y uso de recursos naturales; de Finlandia, en lo relativo al abordaje de los derechos económicos, sociales y culturales, y de Ecuador, respecto a la función social del Estado. Finalmente, en dicha guía, los jóvenes tuvieron la oportunidad de discutir y plantear propuestas para modificar la constitución actual o para el desarrollo de una nueva. 
El diseño original del módulo contempló una séptima clase con presentaciones grupales finales con las propuestas constitucionales del alumnado. Sin embargo, y por los motivos ya señalados, tales presentaciones debieron ser realizadas de forma telemática, mediante videos subidos a una página de Facebook diseñada para tal fin. En ellos, todos los grupos se mostraron proclives a una nueva constitución, en detrimento de modificar la actual, y sus propuestas para esta apuntaron especialmente a fortalecer la función social del Estado, y así garantizar el ejercicio de los derechos económicos, sociales y culturales por parte de la ciudadanía. Asimismo, plantearon la importancia de que una nueva carta fundamental establezca el uso prioritario al servicio de la vida humana de los recursos naturales y bienes de uso público, así como la necesidad de reconocer constitucionalmente a los pueblos originarios de Chile.

Las notas de campo efectuadas apuntan a una fuerte motivación de los partícipes respecto a los temas abordados, de los cuales poseían abundante información previa y, cuando esta les parecía incompleta, recurrían a sus dispositivos móviles para indagar más a fondo. También se expresaron continuamente en las diversas discusiones y cumplieron con entusiasmo y a cabalidad las tareas encomendadas. Por otro lado, se mostraron entusiasmados con salir del aula, aunque en ocasiones fue difícil lidiar con el sol (en la plaza) y con los insectos (en el césped). No obstante, ello abrió la posibilidad de que espontáneamente se integrasen como observadores alumnos de otras carreras, lo cual efectivamente sucedió.

\section{VALORACIÓN DEL MCC POR EL ALUMNADO}

En esta sección se presentan las valoraciones de los jóvenes partícipes respecto al MCC, las cuales incluyen: su interés por el estallido social y el proceso constituyente, su satisfacción con la experiencia didáctica, los aprendizajes que sienten haber desarrollado, las fortalezas y debilidades detectadas, propuestas de mejora y el aporte del módulo a su formación inicial docente. Se trata de resultados obtenidos a partir de la aplicación de cuestionarios, entrevistas y un grupo de discusión.

En los cuestionarios aplicados, un 81\% del alumnado declaró poseer "mucho" o "bastante" interés por el estallido social y el proceso constituyente, mientras que el restante 19\% señaló tener "suficiente" interés. Por su parte, el nivel de satisfacción de la mayoría de los jóvenes con el módulo fue "mucho" (73\%), y en menor medida "bastante" (23\%) y "suficiente" (4\%).

Entre los aprendizajes que el alumnado reconoce haber desarrollado a raíz del MCC se encuentran sobre todo los conocimientos relativos al estallido social -causas y característicasy al proceso constituyente. Asimismo, plantearon que el MCC les permitió profundizar sus conocimientos sobre la Constitución chilena de 1980, así como reconocer las diferencias de esta respecto a constituciones de otros países. Además, se refirieron frecuentemente al desarrollo de habilidades y valores relativos a la comunicación, tales como: pensar de forma más abierta, sostener las ideas con argumentos sólidos, expresarse con claridad y sobre la importancia de respetar las opiniones de otros. La Tabla 3 ofrece mayores detalles respecto a este punto. 


\section{J. J. Salinas et al.}

Tabla 3.Aprendizajes logrados según el alumnado partícipe del MCC.

\begin{tabular}{cc}
\hline Aprendizajes & Referencias \\
\hline Sobre la Constitución chilena de 1980 & $22,8 \%$ \\
\hline Sobre los motivos y características del estallido social y el proceso constituyente & $17,54 \%$ \\
\hline Desarrollo de una forma de pensar más crítica y abierta & $14,03 \%$ \\
\hline Habilidades y valores ligados a la comunicación & $14,03 \%$ \\
\hline Sobre constituciones de otros países y sus diferencias con la de Chile & $12,28 \%$ \\
\hline Desarrollo de la empatía social & $5,26 \%$ \\
\hline Sobre la política y su influencia en la sociedad & $5,26 \%$ \\
\hline Otros & $3,5 \%$ \\
\hline
\end{tabular}

Fuente: Elaboración propia.

Entre las fortalezas del MCC destacadas por el alumnado se repitieron especialmente su abordaje de la contingencia que atravesaba el país en esos momentos y su ambiente abierto, que permitió expresar y debatir las ideas y opiniones libremente, en un ambiente no amenazante y de respeto por el derecho a pensar distinto. Dichas valoraciones fueron constantes en la información recabada. A continuación, algunos ejemplos.

En el grupo de discusión:

Daniel'2: Lo que estaba pasando en el país era como la parte práctica de la asignatura, estaba todo conectado. Lo que pasábamos en clase, salíamos de ella y lo veíamos ahí, in situ. Eso, al menos a mí, me marcó. Nosotros como alumnos no estábamos pensando mucho en matemáticas o en lenguaje, sino básicamente en lo que estaba pasando en ese momento en el país.

Gabriela: Creo que esto nos ayudó a entender que estaba pasando acá, tal vez no muchos teníamos la idea, así «al pan, pan, y al vino, vino» [sin rodeos], de lo que estaba pasando, de todas las injusticias que hay. Y esto, al menos a mí, me ayudó a abrir los ojos, a entender varias cosas.

En cuestionarios:

(...) me ayudó a informarme, porque veía en redes sociales lo que la gente exige en esta revolución. Muchas veces mencionaban el cambio de constitución, pero jamás tuve una idea muy clara de lo que era, mi conocimiento acerca de esto era muy vago. Entonces que se dé esta oportunidad de conocer todo, para entender el contexto en el que se vive, es muy bueno, para así apoyar y tener fundamentos (Isabel).

Porque nos ayudó a todos como curso a poder opinar, sin poner mi opinión sobre otros, a escucharnos y darnos cuenta de que todos tenemos distintas formas de entender un tema (...) (Valeria).

Todos los nombres asignados al alumnado son ficticios. 
En entrevistas:

Pudimos desahogarnos, porque justo trataba de lo que estaba pasando. Entonces como que todos teníamos nuestros puntos y todos queríamos hablar... yo no entendía muy bien que era la constitución y por qué se quiere cambiar, pero en estas clases lo entendí mucho mejor (...) (Claudia).

Las actividades realizadas fueron situadas frecuentemente entre las fortalezas del módulo, siendo calificadas como entretenidas, activas, participativas y capaces de lograr aprendizajes significativos. En este sentido, los jóvenes destacaron que fuesen realizadas grupalmente y fuera del aula, esto último, según señalaron, sería un elemento poco habitual en su formación. De las actividades realizadas en espacios públicos, fue especialmente valorada la oportunidad de discutir la realidad social y política desde una perspectiva calificada por algunos como 'realista', es decir, abierta a la participación (tal como sucedía en los cabildos que se realizaban a lo largo del país), en este caso, de otros estudiantes. Al respecto, un partícipe del grupo de discusión señaló:

Dentro de las actividades realizadas, la que yo más tengo grabada fue en los pastos con los parlantes... tu opinión no solo era escuchada por tus compañeros de curso, que nos escuchamos todos los días, sino que por todo el que estuviese cerca. Todavía recuerdo que había unos compañeros de educación física que estaban muy atentos a lo que estábamos hablando (...) (Daniel).

Resultó notorio que los futuros docentes frecuentemente contrastaron la posibilidad que brindó el MCC para tratar la contingencia en un ambiente de apertura con la falta de oportunidades para hacerlo en su seno familiar, donde tales temas estarían muchas veces vetados como una forma de evitar conflictos. De esta manera, una alumna señaló:

(...) lo bueno fue hablarlo [la contingencia], porque en mi casa esas cosas no se tocan, porque se producen polémicas y peleas. Poder hablarlo y entender cosas fue, al menos para mí, un alivio, porque para mí era un tabú, había muchas cosas de las cuales yo no entendía nada... ayudó mucho escuchar a los compañeros (Gabriela).

En cuanto a las debilidades del MCC, la mayoría de los jóvenes señaló no encontrarlas. Sin embargo, algunos se refirieron a las limitaciones impuestas por el contexto de crisis en que fue realizado y que, finalmente, obligaron a efectuar su última clase de forma online, situación que habría restringido las posibilidades de expresar adecuadamente sus ideas y propuestas en un debate final, actividad respecto a la cual mantuvieron elevadas expectativas que no fueron cumplidas. Por otro lado, también se planteó la dificultad que supone, para realizar actividades de este tipo, la falta de sombra y comodidad en los espacios públicos abiertos de la universidad.

Los veintidós partícipes del MCC consideraron que este fue un aporte a su formación inicial docente, apuntando, en buena parte de los casos, a que dicho módulo fue una experiencia didáctica que les sirve como un ejemplo que podrán considerar situadamente en 


\section{J. J. Salinas et al.}

su futura labor como profesores y profesoras de primaria. Asimismo, fue destacado en este ítem el desarrollo de valores y habilidades de orden comunicativo, incluyendo una mayor apertura a otras ideas. Por otro lado, algunos jóvenes indicaron que el MCC incidió en su forma de ver el aprendizaje y en los fines que asignan a la educación. Sobre estos puntos, se exponen algunas ideas expresadas en los cuestionarios:

Estas actividades las puedo realizar con mis futuros alumnos, ya que es necesario crear una sociedad activa, que puedan lograr un mejor vivir, que tengan conocimiento de lo que pasa en su entorno y lo que puede pasar en un futuro, crear en los alumnos un pensamiento crítico y formarlos para que puedan participar en la sociedad, por esto mismo, para lograr todo esto en mis alumnos, tengo que lograrlo yo principalmente. (Valeria).

Los profesores solemos enseñar como nos enseñaron en la universidad o replicamos la forma en la que hemos aprendido... [me] fue grata esta experiencia didáctica, debido a que somos quienes deben formar a las futuras generaciones (...) (Patricia).

Los jóvenes también plantearon propuestas de mejora para una nueva edición del MCC, entre ellas se cuentan: 1) realizar debates finales, tanto sobre el estallido social como del proceso constituyente; 2) incluir franjas y entrevistas a la ciudadanía en el desarrollo de material audiovisual; 3) hacer llamados abiertos a la comunidad universitaria a participar en las actividades; y 4) trabajar conjuntamente con otros niveles de la misma carrera de PEB y/u otras carreras de la universidad, bajo una lógica interdisciplinar. Respecto a esta última propuesta, el alumnado destacó la ventaja que supondría poner en diálogo una gama más amplia de perspectivas individuales y de lo que denominaron 'la ideología de cada curso y/o carrera', es decir, el conjunto de ideas políticas predominantes en cada uno de estos. Al respecto, en la discusión grupal se propuso:

Gabriela: (...) para la próxima se podría invitar a más gente para ver si piensan como nosotros o distinto, independiente de que carrera sean. Incluso puede ser que nuestra carrera tiene una ideología y ellos otra, y ni siquiera nos estemos dando cuenta. Que hayan escuchado fue bueno, pero me hubiera gustado saber que pensaban de lo que estábamos diciendo.

Daniel: Sería una instancia genial para debatir distintos puntos de vista. Creo que si todas las asignaturas se encaminaran así sacaríamos mejores conclusiones, saldríamos más tolerantes y todos con una mirada mucho más crítica.

Resulta relevante analizar un caso que escapó a la norma respecto al entusiasmo del alumnado con tratar el estallido social en clases. Se trata de Bárbara, quien planteó que, si bien valora positivamente el MCC, discutir sobre tal tema le produjo en ocasiones una sensación de hastío, producto de que este copaba continuamente tanto los medios de comunicación como sus espacios sociales. Al respecto, en su entrevista señaló:

En ese entonces estaba chatísima [cansada] con el tema del estallido social. No quería escuchar nada más al respecto, porque estaba en la U y era estallido social, salía 
de la $\mathrm{U}$ y era estallido social. Iba para todas partes y era estallido social... entonces sentía que era demasiado. Pero siento que es algo personal, no que el estallido sea irrelevante (...).

Dicha joven, no obstante, precisó su acuerdo con tratar temas de actualidad social y política como herramienta didáctica para formar en ciudadanía, sobre lo cual indicó:

Yo creo que está bien, creo que puede ayudar a las personas a abrir su forma de mirar las cosas... cuando discutes con otra persona esta te hace ver que las cosas no son exactamente como tú pensabas o tal vez te hace cuestionar la forma en que miras las cosas. Entonces, creo que es súper importante discutir, sobre todo si son temas relevantes en la vida cotidiana de las personas.

Finalmente, se debe señalar que el análisis por tablas cruzadas de las frecuencias descriptivas, correspondientes a los datos sociodemográficos y las preguntas cerradas del cuestionario aplicado, no arrojó resultados significativos.

\section{DISCUSIÓN DE LOS RESULTADOS}

A partir de los resultados, es posible sostener que tratar el estallido social y el proceso constituyente fue una estrategia didáctica útil para el desarrollo de las competencias ciudadanas del alumnado partícipe, lo cual se condice con posiciones teóricas que apuntan al potencial de los temas candentes y de interés público en la formación de ciudadanos (Magendzo, 2016; Santisteban, 2019) y con el resultado de diversas estudios empíricos realizados tanto en el ámbito de la educación superior (Salinas, 2019, 2020) como en secundaria (Salazar, Orellana \& Arias, 2019; Salinas \& Oller, 2017, 2020). Al igual que en las investigaciones mencionadas, el alumnado demostró poseer conocimientos previos respecto a los asuntos tratados, lo que facilitó su uso con fines educativos.

El MCC supuso el debate de asuntos de interés social en espacios públicos y abiertos de la universidad, en un ambiente de tolerancia y respeto por las diversas ideas, lo cual contribuyó al desarrollo de conocimientos, habilidades y valores ligados a la comunicación, a la participación política y a la comprensión de la propia realidad social. Esto coincide con el planteamiento de Zambrano (2018) quien señala que el estudiante universitario puede desarrollar competencias ciudadanas cuando: “(...) puede manifestar sus emociones, reflexionar sobre sus experiencias vividas, expresar sus puntos de vistas, desarrollar la habilidad comunicativa de la asertividad (...) poner en prácticas [sic] los conocimientos, valores y acciones ciudadanas en el aula" (p. 80).

El alumnado valoró de forma importante la posibilidad de tratar asuntos sociales y políticos de interés público, manifestando en múltiples ocasiones la falta de espacios para tratar dichos temas en el seno familiar. Esto genera preocupación pues, tal como señalan Aguado, Ballesteros, Mata \& Sánchez, (2013): "La familia juega un papel fundamental en el aprendizaje de la práctica ciudadana (...)” (p. 40), y pone de relieve la importancia de abordar 


\section{J. J. Salinas et al.}

la realidad social en la formación en democracia desarrollada en contextos educativos. No obstante, por otro lado, resulta necesario considerar las condiciones de exigencia psicoemocional a la que está sometida la población chilena a raíz del estallido (Madariaga, 2019), por lo que al tratar temas de una connotación pública y una cobertura mediática tan elevadas, especialmente si incluyen violaciones a los DDHH, es necesario escuchar al alumnado y abrir opciones diversas de ser necesario.

Uno de los argumentos usualmente utilizado por las y los partícipes para valorar positivamente el MCC fue que este, a su parecer, constituye una referencia didáctica para desarrollar su propia labor como futuros formadores de ciudadanos. Esto viene a sostener las ideas de autores que advierten del peso que tienen en las prácticas de los docentes sus propias experiencias e ideas previas en el ámbito de la democracia y su ejercicio (Reyes et al. 2013). Ello constituye un motivo más para reforzar el enfoque activo y centrado en el alumnado en la FID, especialmente en materia de ciudadanía donde los estilos tradicionalesexpositivos tienen poco sentido.

En base al interés demostrado por los jóvenes respecto al proceso constituyente, es posible afirmar que la propuesta didáctica realizada contribuyó a empoderarles con el proceso de construir una nueva constitución para el país. A su vez, el fortalecimiento de algunas de sus competencias clave ha contribuido al necesario ejercicio de la ciudadanía que supone dicha tarea. Por tanto, y en el sentido expuesto por Benjumea et al. (2011), se trata de una experiencia que trascendió la lógica instruccional e individual del desarrollo de competencias, propia de la EpC, hacía una experiencia de formación ciudadana fundamentada en un proyecto político de profundización de la democracia.

Durante el MCC, la búsqueda por desarrollar competencias ciudadanas no solo fue un elemento estructurador de aprendizajes concretos, sino que el mismo ejercicio de estas fue clave para establecer una comunidad democrática dotada del ambiente necesario para comunicar desde una estructura dialógica y tolerante. Por ello, es posible sostener la factibilidad del uso del enfoque de 'competencias' en proyectos de formación ciudadana. No obstante, resulta necesario establecer objetivos político-pedagógicos que orienten tanto los aspectos generales de nuestras propuestas educativas como la definición de competencias dirigidas a la profundización de la democracia y la justicia social. Ello permite desarrollarlas considerando las relaciones de poder que están detrás de la configuración de la sociedad y cuya comprensión es imprescindible en la formación de una ciudadanía capaz de transformar su realidad.

\section{CONCLUSIONES}

Los resultados apuntan a un elevado potencial del MCC para el desarrollo de competencias ciudadanas y el empoderamiento constituyente del alumnado partícipe, quienes ejercitaron sus capacidades de pensar críticamente, de comunicar de forma informada y respetuosa, de valorar el derecho de los demás a pensar diferente y de participar activamente en base a un proyecto político para el fortalecimiento de la democracia. Asimismo, demostraron un fuerte interés en el proceso constituyente y por participar en él. 
El alumnado ha valorado la propuesta didáctica de forma positiva, destacando los aprendizajes logrados, tanto en relación al desarrollo de la propia ciudadanía como en lo relativo a su formación inicial docente. Entre los que se cuentan una mejor comprensión de la realidad social del país, del proceso constituyente y del rol de la ciudadanía en este, así como el reconocimiento de la importancia de la formación ciudadana en una sociedad democrática y un mejor entendimiento, por vía del ejemplo directo, de sus características fundamentales.

Respecto a las actividades realizadas, las y los jóvenes resaltaron especialmente la oportunidad de hacer un 'uso público de la razón' en lugares abiertos de la universidad. En este sentido, entre las propuestas de mejora que realizaron destacó la ampliación de la comunidad democrática constituida en el módulo a otros cursos del mismo programa de pedagogía y/o carreras de la universidad, señalando muchos de ellos además su disposición de volver a participar. Se evidencia así una valoración de la experiencia que no solo excede lo instruccional, sino que se manifiesta como un desafío a esquemas de pensamiento predefinidos -entre los que podemos incluir la denominada 'ideología de cada carrera y/o curso'- y como un genuino empoderamiento con la reflexión colectiva respecto al proceso que vive el país y de su rol en él como ciudadanos.

Resulta necesario, en la reedición del MCC, plantear las siguientes mejoras: a) considerar la potencial ansiedad que puede causar en algunos jóvenes tratar temas de alto impacto público y constante difusión mediática, ofreciendo instancias de comunicación entre alumnado y docentes que permitan buscar opciones adecuadas; $b$ ) incluir actividades que permitan ampliar las nociones sobre DDHH, con el objetivo de que estas abarquen sus múltiples generaciones; c) definir espacios públicos, como escenarios del diálogo ciudadano, dotados de condiciones más apropiadas; y d) la ampliación de la 'comunidad democrática' involucrada, a otros cursos y carreras.

El 'Módulo Cabildo Ciudadano' será reaplicado prontamente, mientras el país aún se encuentra en pleno proceso constituyente, ahora considerando las fortalezas, debilidades y propuestas identificadas en este primer ciclo reflexivo. Hoy, el momento histórico que vive Chile reclama más que nunca la participación activa, crítica y empoderada de todas y todos, la formación ciudadana tiene un papel ineludible en dicha tarea.

\section{REFERENCIAS}

Activa Research. (2019). Pulso Ciudadano: Proceso Constituyente. Especial noviembre 2019. [Diapositiva de Power Point]. Activa Research. Recuperado de: https://www.activasite. com/wp-content/uploads/2019/11/Pulso-Ciudadano-Proceso-Constituyente-.pdf

Aguado, T., Ballesteros, B., Mata, P., \& Sánchez, H. (2013). Aprendizaje de la ciudadanía activa: propuestas educativas. En M. Cardona, E. Chiner y A. Giner (Eds.), Investigación e innovación educativa al servicio de las instituciones y comunidades globales, plurales $y$ diversas (pp. 35-42). Alicante: Universidad de Alicante.

Aniñir, D., \& Candina, A. (2020). Estallido social: elementos para una genealogía de las violencias. Meridional. Revista Chilena de Estudios Latinoamericanos, 14, 241-246. Recuperado de https://meridional.uchile.cl/index.php/MRD/article/view/57136 


\section{J. J. Salinas et al.}

Barton, K. C., \& McCully, A. W. (2007). Teaching Controversial Issues.... where controversial issues really matter. Teaching History, 127, 13-19. Recuperado de: https://www.history. org.uk/secondary/resource/700/teaching-controversial-issueswhere-controversia

Bassa, J. (2020). Chile decide por una nueva constitución. Santiago de Chile: Planeta.

Benjumea, M., Gutiérrez, A., Jaramillo, O., Mesa, A., \& Pimienta, A. (2011). Formación Ciudadana (FC) y Educación para la Ciudadanía (EpC). Aproximaciones conceptuales y mínimos compartidos. Revista Temas, 5, 211-224. http://doi.org/10.15332/rt.v0i5.695

CADEM. (2020). Encuesta Plaza Pública. Cuarta semana de julio. Estudio $N^{\circ} 341$.

Recuperado de: https://plazapublica.cl/wp-content/uploads/2020/07/Track-PP-341Julio-S4.pdf

Castellví, J. (2019). Literacitat Crítica Digital en els Estudis Socials. Estudis de Cas en Educació Primària [Dissertation]. Barcelona: Universitat Autònoma de Barcelona. Recuperado de: https://www.tdx.cat/handle/10803/669394

Castellví, J., \& González-Monfort, N. (2020). Educación para una ciudadanía crítica en España: cambios y continuidades. (Educação para uma cidadania crítica na Espanha: mudanças e continuidades). Revista Espaço do curriculo, 13(2), 166-175.

Castellví, J., Massip, M., \& Pagès, J. (2019). Emociones y pensamiento crítico en la era digital: un estudio con alumnado de formación inicial. Revista de Investigación en Didáctica de las Ciencias Sociales, 5, 23-41. http://doi.org/10.17398/2531-0968.05.23

Council of Europe. (2018). Reference framework of competences for democratic culture. Descriptors of competence for democratic culture (Vol. 2). Strasbourg: Council of Europe Publishing.

Estellés, M., \& Castellví, J. (2020). The Educational Implications of Populism, Emotions and Digital Hate Speech: A Dialogue with Scholars from Canada, Chile, Spain, the UK, and the US. Sustainability, 12(15), 6034. http://doi.org/10.3390/su12156034

Flores, L., \& García, C. (2011). Los desafíos de la formación ciudadana y la cohesión social frente a la des-subjetivación del sistema. Hacia una interpretación del fenómeno social desde la subjetividad. Estudios Pedagógicos, 37(2), 329-344. http://doi.org/10.4067/ S0718-07052011000200020

Freire, P. (1997). La educación como práctica de la libertad (45a ed.). Madrid: Siglo XXI.

Freire, P. (1999). Política y educación ( $2^{\mathrm{a}}$ ed.). Ciudad de México: Siglo XXI.

Fuentes, R. (2019, noviembre 7). Cabildos autoconvocados: 15 mil personas se han sumado a la iniciativa popular en todo el país. Radio Universidad de Chile. Recuperado de: https://radio.uchile.cl/2019/11/07/cabildos-autoconvocados-15-mil-personas-se-hansumado-a-la-iniciativa-popular-en-todo-el-pais/

Giroux, H. (1997). Los profesores como intelectuales. Hacia una pedagogía crítica del aprendizaje. Barcelona: Paidós.

Giroux, H. (2004). Teoría y resistencia en educación. Una pedagogía para la oposición (6ª ed.). Ciudad de México: Siglo XXI.

Grez, S. (2019). Rebelión popular y proceso constituyente en Chile. En M. Folchi (Ed.), Chile despertó. Lecturas desde la Historia del estallido social de octubre (pp. 13-20). Santiago de Chile: RedesTd. 
Heiss, C. (2020). ¿Por qué necesitamos una nueva constitución? ( $2^{\mathrm{a}}$ ed.). Santiago de Chile: Aguilar.

Hernández, R., \& Mendoza, C. (2018). Metodología de la investigación. Las rutas cuantitativa, cualitativa y mixta. Ciudad de México: McGraw Hill.

Hoecker, G., \& Dardel, M. (Eds.). (2019). Chile Despertó. Cuaderno pedagógico. Recuperado de: https://cuadernochiledespertoschool.files.wordpress.com/2019/11/cuaderno-chiledespertocc81-ediciocc81n-1-completa-corregida.pdf

Instituto nacional de Derechos Humanos. (2020). Reporte general de datos sobre violaciones a los derechos humanos. 19 de marzo de 2020. Recuperado de: https://www.indh.cl/bb/ wp-content/uploads/2020/04/Reporte-INDH-19-de-marzo-de-2020.pdf

Kennedy, K. J. (2019). Civic and Citizenship Education in Volatile Times. Preparing Students for Citizenship in the 21st Century. Springer.

Latorre, A. (2016). La investigación-acción. En R. Bisquerra (Ed.), Metodología de la investigación educativa (5a ed, pp. 361-386). Madrid: La Muralla.

Legardez, A., \& Simonneaux, L. (2006). L'école à l'épreuve de l'actualité. Enseigner les questions vives. Paris: ESF editeur.

Madariaga, C. (2019). El "Estallido social" y la salud mental de la ciudadanía: Una apreciación desde la experiencia PRAIS. Revista Chilena de Salud Pública, 23(2), 146-156. http://doi. org/10.5354/0719-5281.2019.56475

Magendzo, A. (2016). Incorporando la perspectiva controversial en el curriculum disciplinario. Revista Iberoamericana de Educación Superior, 7(19), 118-130. http://doi.org/10.22201/ iisue.20072872e.2016.19.190

Massot, I., Dorio, I., \& Sabarriego, M. (2016). Estrategias de recogida y análisis de la información. En Bisquerra, R. (Ed.), Metodología de la investigación educativa (pp. 321-357). Madrid: La Muralla.

Mesa, A., \& Benjumea, M. (2011). La educación para la ciudadanía en la educación superior. Unipluriversidad, 11(1), 1-12. Recuperado de: https://revistas.udea.edu.co/index.php/ unip/article/view/10581/9712

Morán, J. M. (2019). Chile despertó: el modelo chileno, la matriz de desigualdad y la protesta de 2019. Crítica y Resistencias. Revista de Conflictos Sociales Latinoamericanos, 9(9), 5469. Recuperado de: https://www.criticayresistencias.com.ar/index.php/revista/article/ view/120

Morillas, M. (2006). Competencias para la ciudadanía. Reflexión, decisión, acción. Madrid: Narcea.

Mujica, P. (2019). ¿Qué puede cambiar una nueva constitución? EducarChile. Recuperado de: https://www.educarchile.cl/sites/default/files/2019-11/Qué-puede-cambiar-unanueva-Constitución.pdf

OREALC/UNESCO. (2017). La formación inicial docente en educación para la ciudadanía en América Latina. Análisis comparado de seis casos nacionales. Recuperado de: http:// educacion.udp.cl/wp-content/uploads/2017/03/DOC-2-DOCENTES_Y_CIUDADANIAESP-FINAL-1.pdf

Ortuño, J., Gómez, C., \& Ortiz, E. (2012). La evaluación de la competencia educativa social 


\section{J. J. SAlinas et al.}

y ciudadana desde la didáctica de las ciencias sociales. Un estado de la cuestión. Didáctica de las Ciencias Experimentales y Sociales, 26, 53-72. http://doi.org/10.7203/ dces.26.1931

Repetto, A. (2016). Crecimiento, pobreza y desigualdad: la vía chilena. Economía y Política, 3(1), 71-101. Recuperado de: http://www.economiaypolitica.cl/index.php/eyp/article/ view/29

Reyes, L., Campos, J., Osandón, L., \& Muñoz, C. (2013). El profesorado y su rol en la formación de los nuevos ciudadanos: desfases entre las comprensiones, las actuaciones y las expectativas. Estudios Pedagógicos, 39(1), 217-237. http://doi.org/10.4067/S071807052013000100013

Sáez-Rosenkranz, I. (2019). \#Chiledespertó. Guía del profesorado y materiales del estudiante. Universitat de Barcelona. Recuperado de: https://pcriticoycssoc.wixsite.com/ chiledesperto

Salazar, G. (2020). Acción Constituyente. Un texto ciudadano y dos ensayos históricos. Santiago de Chile: Tajamar Editores.

Salazar, R., Orellana, C., \& Arias, Á. (2019). Temas controversiales como herramienta en la formación ciudadana: una propuesta didáctica para el tratamiento de la distribución de las riquezas. En C. Muñoz y B. Torres (Eds.), Escuela y Formación Ciudadana: temas, escenarios y propuestas para su desarrollo (pp. 119-140). Concepción: Editorial Universidad de Concepción.

Salinas, J. (2019). El Museo Regional de Antofagasta. Un recurso didáctico para la formación inicial docente. En M. Hortas y A. Dias (Eds.), Enseñar y aprender didáctica de las ciencias sociales: la formación del profesorado desde una perspectiva sociocrítica (pp. 650-660). Lisboa: AUPDCS - ESE/PL. Recuperado de:

https://www.researchgate.net/publication/332762485_El_Museo_Regional_de_Antofagasta_ Un_recurso_didactico_para_la_formacion_inicial_docente

Salinas, J. (2020). Guías Turísticos Patrimoniales. Formando futuros docentes en el centro de la ciudad de Antofagasta. En P. Ramírez (Ed.), Experiencias Educativas Patrimoniales (pp. 14-17). Antofagasta: Corporación PROA. Recuperado de:

https://www.researchgate.net/publication/343323594_Guias_Turisticos_Patrimoniales_ Formando_futuros_docentes_en_el_centro_de_la_ciudad_de_Antofagasta

Salinas, J., \& Oller, M. (2017). Debatiendo temas controversiales para formar ciudadanos. Una experiencia con alumnos de secundaria. Práxis Educativa, XXI(3), 40-48. http://doi. org/10.19137/praxiseducativa-2017-210305

Salinas, J., \& Oller, M. (2020). Formar ciudadanos a través de la acción sobre los problemas sociales de la comunidad. Praxis Educativa, 24(1), 1-14. Recuperado de: https://cerac. unlpam.edu.ar/index.php/praxis/article/view/3540

Santisteban, A. (2019). La enseñanza de las ciencias sociales a partir de problemas sociales o temas controvertidos: estado de la cuestión y resultado de una investigación. El Futuro del Pasado, 10, 57-79. http://doi.org/10.14516/fdp.2019.010.001.002

Santisteban, A., \& Pagès, J. (2007). El marco teórico para el desarrollo conceptual de la Educación para la Ciudadanía. En J. Pagès, y A. Santisteban (coords.). Educación para la 
ciudadanía (pp. 1-14). Madrid: Wolters Kluwer.

Thornton, S. (1991) Teacher as curricular-instructional gatekeeper in Social Studies. In Shaver, J. P. (edit.), Handbook of Research on Social Studies teaching and learning. A project of The National Council for the Social Studies (pp. 237-248). New York: Macmillan.

Weiner, E. (2008). Pedagogía Crítica y la crisis de la imaginación. En P. McLaren y J. Kincheloe (Eds.), Pedagogía Crítica. De qué hablamos, dónde estamos (pp. 89-116). Barcelona: Graó.

Westheimer, J., \& Kahne, J. (2004). What kind of citizen? The politics of educating for democracy. American Educational Research Journal, 41, 237-269. http://doi. org/10.3102/00028312041002237

Zambrano, E. (2018). Prácticas pedagógicas para el desarrollo de competencias ciudadanas. Revista Electrónica de Investigación Educativa, 20(1), 69-82. http://doi.org/10.24320/ redie.2018.20.1.1409 